\title{
Explorative study on the aftercare of pediatric brain tumor survivors: a parents' perspective
}

\author{
Eline J. Aukema • Bob F. Last • \\ A. Y. Netteke Schouten-van Meeteren • \\ Martha A. Grootenhuis
}

Received: 10 February 2010 / Accepted: 23 August 2010 /Published online: 10 September 2010

(C) The Author(s) 2010. This article is published with open access at Springerlink.com

\begin{abstract}
Purpose Whilst the need for aftercare for long-term sequelae of brain tumor survivors is well known and evident, information from a parent's perspective is lacking on whether the need for aftercare is detected in time, and whether the aftercare is timely initiated and meets the needs for aftercare. Methods A survey regarding aftercare in five domains of long-term sequelae (neurocognitive, physical, emotional, social and parenting problems) was sent to 57 parents of survivors treated for a brain tumor in our center.

Results Forty-two (74\%) parents participated in this study. With a mean period of 8.1 years $(\mathrm{SD}=3.9)$ since start of treatment, the majority of the survivors (mean age $=$ 14.7 years, $\mathrm{SD}=3.8$ ) needed aftercare in several domains of functioning. This need was highest and most met for physical sequelae $(N=34)$, and lowest but still substantial and least met for parental difficulties $(N=11)$. Parents of
\end{abstract}

\section{E. J. Aukema • B. F. Last • M. A. Grootenhuis}

Psychosocial Department, Emma Children's Hospital/Academic

Medical Center (AMC), University of Amsterdam,

Amsterdam, The Netherlands

B. F. Last

Department of Developmental Psychology,

Free University of Amsterdam,

Amsterdam, The Netherlands

A. Y. N. Schouten-van Meeteren

Department of Pediatric Oncology, Emma Children's Hospital/ Academic Medical Center (AMC), University of Amsterdam, Amsterdam, The Netherlands

E. J. Aukema $(\bowtie)$

Psychosocial Department, Emma Children's Hospital,

(Room G8-224), AMC,

Meibergdreef 9 ,

1105 AZ Amsterdam, The Netherlands

e-mail: e.j.aukema@amc.nl survivors with surgery only as treatment reported a similar need for aftercare as those of survivors with adjuvant therapy. Most of the survivors received aftercare; however, substantial delay of aftercare and self-referral for aftercare were frequently reported. Furthermore, parents showed a lack of knowledge about and use of aftercare services.

Conclusions Increased awareness for the need for psychosocial aftercare is required. Coaching, psycho-educative programs about coping with the long-term sequelae and information about available specialized aftercare services are required to meet the needs of brain tumor survivors and their parents more adequately.

Keywords Children · Brain tumors · Late effects · Follow-up $\cdot$ Aftercare

\section{Introduction}

The overall survival rate of children with a brain tumor has increased substantially due to multimodal treatment strategies [1]. As a consequence of the tumor and its multimodal treatment, survivors of childhood brain tumors are at considerable risk for a variety of long-term sequelae. The current literature comprehensively outlines the multiple neurocognitive difficulties [2-4], problems in physical functioning [5, 6] and psychosocial difficulties [7-11] experienced by survivors of childhood brain tumors and the impact on family functioning [12, 14]. There is growing evidence that not only survivors treated with cranio(spinal) radiotherapy, but also survivors of low grade brain tumors treated with surgery only, experience several long-term sequelae $[15,16]$.

The group of brain tumor survivors is growing and results in an increased demand for structured aftercare for 
which multidisciplinary long-term follow-up programs (e.g. $[10,17-20])$ are desirable. For example, Wallace et al. [20] proposed a three-level follow-up model to organize the intensity and frequency of the follow-up care. Recently, Bowers et al. [21] evaluated the use of these long-term follow-up programs in different centers from the Children's Oncology Group (COG) group members and found considerable variations in follow-up care in different centers despite these guidelines. They reported multiple barriers in the delivery of follow-up care, barriers in finance and insurance, but also more intrapersonal barriers knowing patients' uncertainty about the need to follow-up care and the desire not to be followed in a pediatric aftercare program.

Variation in brain tumors and treatment modalities may influence the amount, interval and length of the follow-up care based on health risks. More knowledge about the necessary follow-up care for children who had surgery only as treatment for their brain tumor compared to those treated with adjuvant therapy is needed. The follow-up care can vary in the degree of care provided. This could be caused by either insufficient awareness of long-term sequelae or due to restrictions in multidisciplinary care for instance a separate neurosurgery department. Research so far indicates that timely intervention and preventive programs improve different aspects of quality of life of survivors of childhood cancer. Recently, several evidence-based interventions became available, including cognitive remediation [22-24] and emotional and social programs [25-27]. By remediating cognitive functions and offering early interventions at school (e.g. extra tutoring, audio books, use of a laptop) new learning facilities are provided, improving future perspective and psychosocial functioning of these survivors.

Although the need for aftercare for sequelae of some survivors are obvious, especially for children treated with adjuvant therapy, information is lacking on whether this need is detected in time by professionals and whether existing aftercare is timely initiated. Furthermore because of the long lasting existence of the sequelae and the risk for these survivors to grow further into their deficits [16], it is essential that parents and survivors are well informed about the possible sequelae. Thereby they should have knowledge about available aftercare services. Little is known about the parents' perspective on the follow-up aftercare; such as whether their own needs for aftercare for their child are met, whether parents have enough knowledge of and make use of aftercare services available or whether parents experience certain barriers in the care trajectory after the treatment.

With a survey we retrieved information from parents of a diverse group of brain tumor survivors in order to gain insight in the needs for aftercare, the course of aftercare and needed improvements regarding the provided aftercare.
The aims of this explorative study were to: (1) describe the need for aftercare for long-term sequelae perceived by parents and to explore the aftercare, (2) analyze differences in the need for aftercare in children treated with or without adjuvant therapy and (3) evaluate parents' knowledge about and use of aftercare services available in the Netherlands.

\section{Methods}

Procedure

Parents of all children who had completed treatment for a brain tumor between 1990 and 2006 at the Emma Children's Hospital Academic Medical Center, Amsterdam, The Netherlands $(N=57)$, were invited to participate in the study. Children with neurofibromatosis were excluded because of their specific cluster of impairments.

The inclusion criteria were: (1) treated for a brain tumor of any histology diagnosed between the ages of 0 and 18 , (2) current age of the survivor between 4 and 20 years, (3) complete remission or stable residual tumor, (4) at least 1 year after the end of treatment and (5) able to complete a questionnaire in Dutch. Parents of survivors who met the inclusion criteria in May 2007 were invited to this study, which was approved by the local medical ethics committee.

Parents were firstly contacted by phone and then received an introductory letter explaining the aim of the study and asking for their participation. The letter was accompanied by questionnaires including a survey about the aftercare, as reported in this article, an informed consent form, and a stamped return envelope. Each family received one packet of questionnaires by mail to be completed at home. Non-responders were contacted by phone and a reminder letter was sent to increase response.

\section{Measures}

The survey with eight questions per domain of child functioning and parenting includes both pre-coded as open questions about the need for aftercare, the type of provided aftercare, timing and referral, needs for improvements and knowledge about and use of aftercare services. The choices of the specific domains of child functioning and parenting were based on the known long-term sequelae of childhood cancer survivors [2-16] and aftercare services available in the Netherlands. The questions were formulated by several experienced oncologists and psycho-oncologists of our hospital.

Answering options with service examples were given as well as space to write down other options. Afterwards, answers were categorized in the categories described below. 
First, the prevalence and type of long-term sequelae in five different domains and the need for aftercare were evaluated among the parents. The domains covered different types of long-term sequelae, classified as follows: (1) neurocognitive problems (regarding attention, memory, processing speed, executive function, visual perceptual motor skills and fine motor skills); (2) physical problems (regarding energy level [e.g. headache, fatigue], hormone function [e.g. early puberty, growth retardation], motor function [e.g., fine and gross dexterity, balance, strength], epilepsy, visual and auditory function); (3) emotional problems (including internalizing [fearfulness, worrying, difficulties in expressing emotions], externalizing [frustration, anger] illness experience [difficulties with the disease, feeling different] and self-esteem); (4) social problems (regarding social isolation [e.g. contact making, maintain relationships], adult-centered, being bullied and quarreling with peers); and (5) parenting problems (including autonomy encouragement, differences in parenting style, overprotection and overindulgent behavior).

Based on these answers, a mean score of need for care in the different domains (0-5) was calculated per participant.

Second, the type of aftercare as provided was asked, categorized in specific answer options and examples of aftercare services available in the Netherlands for each domain. These were, for neurocognitive problems: educational support at a regular school, special needs school, neurocognitive test administration and/or remediation; for physical problems: physiotherapy and/or occupational therapy, medication, specific diet, motor support equipment (e.g. motor aids, adjusted sport participation), visual support (e.g. eyeglasses or training to cope with visual deficits), auditory support and special education needs based on motor dysfunction; for emotional problems: therapy from a psychologist, other forms of supportive care (e.g. homeopathy, creative therapy), parental support and contact with fellow patients; for social problems: therapy from a psychologist, social skills training, parental support and contact with fellow patients; and for parenting problems: therapy from a psychologist or another professional giving parenting support and contact with fellow parents. We evaluated the aftercare in terms of the referrer and need for improvements regarding (1) timely response to the need for aftercare, (2) psycho-education and support in finding aftercare services, (3) proactive approach from school and (4) proactive screening of needs for aftercare. To explore who referred participants to the aftercare, we created the following categories: hospital staff (doctors, psychologists or other caregivers from the hospital), school staff (teachers or other staff members of the school) and parents themselves (whether or not in cooperation with the general practitioner).

Finally, the knowledge of the existence and use of different aftercare institutions and websites in The Netherlands were asked. The knowledge was categorized in websites with psycho-education about acquired brain injury and brain tumors, institutions for aftercare services (rehabilitation centers, hospitals), parents' association for children with cancer and family camps, and school services (websites with school aids, information and school liaisons).

Individual patient data regarding gender, date of birth and medical data regarding date of primary diagnosis, histological type of tumor, location of tumor, type of treatment, secondary operations (including progression and relapse) and duration of adjuvant treatment were gathered from the medical files.

\section{Statistical analyses}

The participants and non-participants were compared with respect to demographic and medical variables using Independent Samples $t$-tests and the chi square $\left(\chi^{2}\right)$ tests $(p<0.05)$. Frequency analyses were conducted to assess the prevalence and kind of parent-reported long-term sequelae, the need for aftercare and the amount of domains for which aftercare was needed (0-5) was calculated. Then, associations between need for aftercare and treatment ('surgery only' and 'surgery and adjuvant therapy ') were examined with $\chi^{2}$ tests at $p<0.05$ and with Mann-Whitney $U$-tests, differences in the amount of need for care in different domains (0-5) between these two groups were examined at $p<0.05$. Also, frequency analyses were used to evaluate the provided aftercare and the familiarity with websites and institutions. The Statistical Package for Social Sciences (SPSS) version 16 was used for all analyses.

\section{Results}

Participants

Forty-two (74\%) of the 57 parents participated in the study and returned their completed survey. Characteristics of the participants and non-participants are presented in Table 1. Half of the participants were treated with surgery plus adjuvant therapy. Non-participants did not differ from participants in demographic and medical variables. The reasons given for not participating were lack of interest or no time to fill in the questionnaires.

Needs for and exploration of the aftercare in different domains of long-term sequelae

The need for aftercare and received aftercare and needs for improvements with the aftercare trajectory are presented in Tables 2 and 3.

Overall, most parents reported need for aftercare in several domains: $17 \%$ reported problems in two domains, 
Table 1 Characteristics of the (non-)participants $(N=57)$

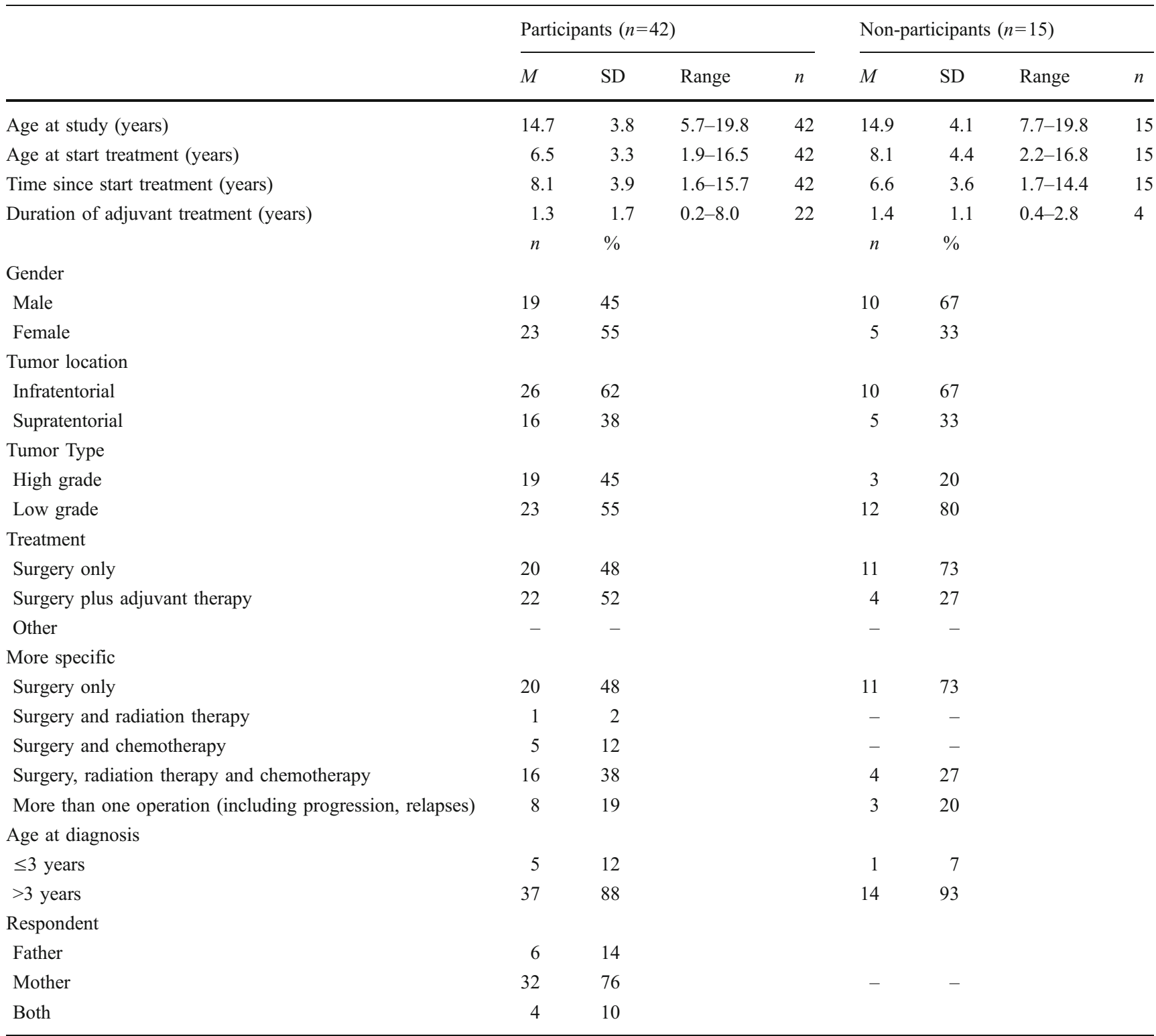

$29 \%$ reported problems in three domains, $19 \%$ reported problems in four domains and $14 \%$ reported problems in all domains. Overall, the mean amount of domains for which aftercare was needed according to the parents was 2.8 (median $=3, \mathrm{SD}=1.4$ ).

\section{Neurocognitive domain}

Two thirds $(N=31 ; 74 \%)$ of the parents reported neurocognitive problems, most often consisting of problems with attention, processing speed and fine motor skills. Thirty of these survivors $(97 \%)$ needed aftercare; 28 survivors received specialized aftercare, two survivors did not receive the care they needed. Aftercare was comprised of use of special educational services knowing remedial teaching at a regular school or transition to a school for special education and neuropsychological testing with or without remediation.

Parents reported that the hospital staff, school and parents themselves equally acted as referral sources for specific aftercare. Half of the parents expressed their needs for improvements regarding a sooner response to the need for aftercare and more support and psycho-education in general. In particular, they reported that school should point out problems more actively.

\section{Physical domain}

Survivors with physical problems $(N=38,90 \%)$ covered motor dysfunctions, such as fine and gross dexterity and balance, being tired and having headaches, as well as early 


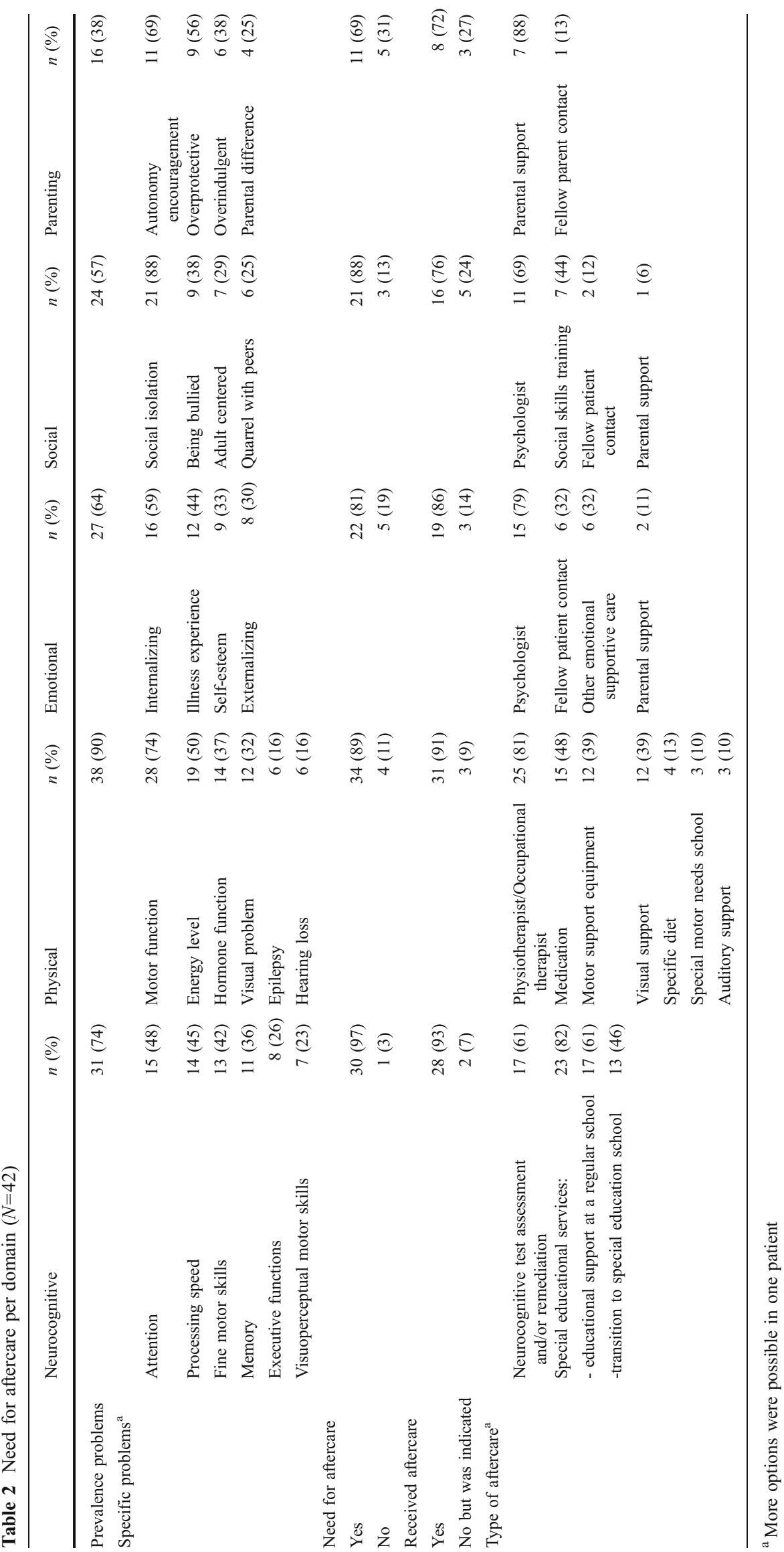


Table 3 Evaluation of the received aftercare in different domains

\begin{tabular}{|c|c|c|c|c|c|c|c|c|c|}
\hline & Neurocognitive & $n(\%)$ & Physical & $n(\%)$ & Emotional & $n(\%)$ & Social & $n(\%)$ & Parenting $n(\%)$ \\
\hline \multicolumn{10}{|l|}{ Need for aftercare } \\
\hline Yes & & $30(97)$ & & $34(89)$ & & $22(81)$ & & $22(88)$ & $11(69)$ \\
\hline \multicolumn{10}{|l|}{ Received aftercare } \\
\hline Yes & & $28(93)$ & & $31(91)$ & & $19(86)$ & & $17(77)$ & $8(73)$ \\
\hline No but was indicated & & $2(7)$ & & $3(9)$ & & $3(14)$ & & $5(23)$ & $3(27)$ \\
\hline \multicolumn{10}{|l|}{ Referrer $^{\mathrm{a}}$} \\
\hline Hospital staff & & $10(36)$ & & $29(85)$ & & $8(42)$ & & $2(17)$ & $1(13)$ \\
\hline Schoolteacher or staff & & $9(32)$ & & $5(15)$ & & $3(16)$ & & $6(35)$ & $1(13)$ \\
\hline Parents themselves & & $10(36)$ & & $4(12)$ & & $8(42)$ & & $9(53)$ & $4(50)$ \\
\hline Not specified & & $2(7)$ & & $2(6)$ & & $4(21)$ & & $2(12)$ & $2(25)$ \\
\hline Need for improvements ${ }^{\mathrm{a}}$ & & $17(57)$ & & $11(31)$ & & $11(50)$ & & $12(57)$ & $7(64)$ \\
\hline \multicolumn{10}{|l|}{ More specific } \\
\hline Timely response to need for care & & $13(43)$ & & $9(27)$ & & $7(32)$ & & $8(36)$ & $5(45)$ \\
\hline $\begin{array}{l}\text { Psycho-education about possible } \\
\text { long-term sequelae and available } \\
\text { aftercare services and support in } \\
\text { finding aftercare service }\end{array}$ & & $11(37)$ & & $6(18)$ & & $9(41)$ & & $5(23)$ & $5(45)$ \\
\hline Proactive approach from school & & $6(20)$ & & $2(6)$ & & $1(5)$ & & $1(5)$ & - \\
\hline $\begin{array}{l}\text { Proactive screening of needs for } \\
\text { aftercare }\end{array}$ & & $7(23)$ & & $3(9)$ & & $4(18)$ & & $6(27)$ & $3(27)$ \\
\hline
\end{tabular}

${ }^{\text {a }}$ More options were possible in one patient

puberty and delayed growth. Most of these survivors $(N=$ 34, 89\%) needed professional aftercare; 31 survivors received specialized aftercare; three did not receive aftercare though it was needed.

Most survivors received aftercare from a physiotherapist and/or occupational therapist, and received medication (anti-epileptic drugs and/or hormones) and motor or visual support equipment.

In most cases, the hospital staff referred participants to the particular specialist or therapist. A third of the parents had suggestions for improvements regarding the timing and the need for more support and information about getting the needed aftercare.

\section{Emotional domain}

Twenty-seven parents $(64 \%)$ reported emotional problems in their child, especially internalizing symptoms regarding illness experiences and self-esteem. The majority of these survivors needed aftercare $(N=22,81 \%) ; 19$ survivors received aftercare; three did not receive the care that was needed. The aftercare given included treatment by a psychologist, other emotional supportive care and fellow patient contact.

The hospital staff and parents initiated this type of aftercare. Needs for improvement were expressed by half of the parents. More specifically, parents asked for more support and psycho-education on the possible emotional long-term sequelae, asked for information on aftercare available and asked for a more timely response on emotional problems.

\section{Social domain}

Social problems were reported in 24 survivors $(57 \%)$ and involved social isolation, being bullied and adult-centered behavior. The majority of these survivors $(N=21,88 \%)$ needed aftercare; 16 received specialized aftercare, and five did not receive aftercare although the need existed according to the parents.

Aftercare was primarily comprised of treatment by a psychologist or a social skills training. In almost half of the patients the care was initiated by the parents themselves, followed by school and the hospital staff. Parents expressed their need for improvements regarding a proactive screening for social problems in general and thereby a sooner referral for specific aftercare. Parents also wanted to receive more information about possible difficulties that could arise in the child's social behavior.

\section{Parenting domain}

A third of the parents $(N=16,38 \%)$ reported problems with encouraging autonomy in their child and being overprotec- 
tive and overindulgent to the child. Eleven (69\%) needed extra care, of which eight received aftercare and three did not get the needed aftercare. Mostly, aftercare was comprised of professional parental support as was initiated by parents themselves.

More than half of the parents reported that improvements were needed, in particular more information about possible parenting difficulties and better screening and, if necessary, referral for these difficulties.

\section{Associations and differences between need for aftercare and type of treatment}

No significant associations were found between the need for aftercare in the different domains and treatment modalities.

The groups also did not differ in the mean amount of domains for which aftercare was needed (mean amount of domains in 'surgery only' group $=2.7$ and in 'surgery plus adjuvant therapy' group=3.0 domains; $U=189, p=0.4$ ).

\section{Knowledge about and use of relevant institutions and websites}

The familiarity and use of relevant institutions and websites for support and interventions in case of long-term sequelae are reported in Table 4.

Parents were most familiar with the aftercare services in the hospital (81\%), the parents' association of children with cancer and camps for survivors $(69 \%)$ and websites with psycho-education about required brain injury and brain tumors $(64 \%)$. They were least familiar with educational services and special educational aids (31\%).

Despite the knowledge of relevant institutions and websites, only $45 \%$ of parents actually made use of institutional aftercare, followed by the parents' association of children with cancer and camps for survivors (38\%), use of websites $(19 \%)$ and finally school services $(10 \%)$.

\section{Discussion}

Despite the fact that childhood brain tumor survivors are at considerable risk for long-term sequelae for which aftercare is needed, little is known about the recognition of this need for aftercare and whether the aftercare meets the needs of the parents of this group of survivors.

Our explorative study from a parents' perspective highlights a considerable need for aftercare, not only for physical and neurocognitive problems, but also a substantial need for social, emotional and parenting aftercare. These findings are comparable with the outcome of former studies [2-6]. This need for aftercare has been expressed by the parents regardless of whether survivors had received adjuvant therapy, which is in line with the findings in earlier studies $[15,16]$.

Remarkably, our survey reveals that despite increased awareness of long-term sequelae among professionals, parents reported considerable delays in identification of the sequelae, and that more timely reactions to the needs for aftercare are required in particular for psychosocial problems resulting from disease and treatment.

Furthermore, parental knowledge about aftercare services is lacking, indicating a necessity for a timely and repeated screening of long-term sequelae that should result in providing specialized aftercare and psycho-education to all brain tumor survivors.

\section{Evaluation of the aftercare}

Although parents reported that most survivors received specialized aftercare when needed, a substantial number of parents expressed the need for improvements regarding the aftercare trajectory. The need for aftercare was most unmet for parenting problems, and most met for neurocognitive problems. For the psychosocial domains and parenting domain, more than half of the parents reported that improvements were needed regarding the timing of the response to the need for aftercare, more psycho-education and support in finding the aftercare services and a proactive approach of the needs.

More specific, a timely response to the need for aftercare and timely referral was highest for physical aftercare, medium for emotional, social and neurocognitive aftercare, and lowest for parenting aftercare. Parents often had to refer their child for psychosocial aftercare themselves and the timing of the referral by professionals was often perceived

Table 4 Knowledge about and use of relevant institutions and websites

\begin{tabular}{|c|c|c|c|c|c|c|}
\hline & Familiar with & $n$ & $\%$ & Make use of & $n$ & $\%$ \\
\hline Websites with psycho-education about acquired brain injury and brain tumors & & 27 & 64 & & 8 & 19 \\
\hline Institutions for aftercare services (rehabilitation centers, hospitals) & & 34 & 81 & & 19 & 45 \\
\hline Parents' association for children with cancer and family camps & & 29 & 69 & & 16 & 38 \\
\hline School services (websites with school aids, information and school liaisons) & & 13 & 31 & & 4 & 10 \\
\hline
\end{tabular}

More options were possible in one patient 
as too late. In addition, more psycho-education about psychosocial long-term sequelae and support in finding aftercare services and a proactive screening of needs for aftercare were suggestions to improve the aftercare for psychosocial and parenting problems. A proactive approach from the school (liaison) could be helpful in identifying difficulties and could lead to a timely intervention, especially for learning difficulties. From this, we conclude that the aftercare providers respond to physical problems appropriately, while more awareness is needed for aftercare for psychosocial problems and parenting difficulties. This finding is consistent with literature which indicates that psychological aftercare is needed. Eiser [10] stated that psychological care of survivors needs to be integrated in the routine assessment. Recklitis et al. [28] also points to the need to provide routine psychological assessment in addition to screening for physical problems.

Failure to detect and thereby meet the needs for aftercare timely could be explained from the perspective of several barriers. First of all, specific knowledge about late sequelae from childhood brain tumors must be well communicated with the parents. Second, it could be the case that doctors did not respond adequately to the needs of parents. Poor doctor-patient communication and contact can be viewed as an interpersonal barrier. It is known that the quality of the consult is determined by the quality of the doctorpatient relationship, which is predictive for compliance with the doctor's advice [29].

Moreover, intrapersonal barriers in parents could also have led to delayed referral and in delayed use of the existing and familiar aftercare services. Parents may have underreported the existing problems in their child for a while in order to maintain a high degree of optimism. This behaviour, which may be viewed as denial, could also be viewed as 'selective information processing' [30]. In addition, looking to the parental reports we consider that at least a substantial part of delay in aftercare has been caused by a lack of urgency of the problems from a parent's perspective in our survivor group. The fact that a substantial part of the parents are familiar with aftercare services but only a few use these services seems indicative for this delay. Therefore, parents could underreport the existing problems through lack of energy to be confronted with new problems.

Overall, half of the parents showed a lack of knowledge about long-term aftercare services, which shows a barrier in the prevention of future problems in this survivor group at risk.

Interestingly, Oeffinger and Wallace [31] described several potential tools in overcoming some of the barriers mentioned before by introducing a Passport for Care, a web-based program with individualized summary of the patients history combined with the COG long-term follow- up guideline. Also, our results support recommendations from Hewitt et al. [32] for a survivorship care plan which addresses psycho-education about long-term aftercare and psychosocial support services available. Online psychoeducation and recording the consult might improve the accessibility of the necessary information [33].

\section{Parenting problems}

In our group of survivors, one third of the parents reported parenting problems, including difficulties with encouraging autonomy in their child and being overprotective. These problems seem to have remained unnoticed by professionals at school and the hospital staff. From this, we conclude, consistent with literature [12-14], that more attention is needed to estimate the burden and needs of parents. Coaching parents in coping with their children's disabilities might reduce parental stress and empower their parenting style in everyday life [34]. It is known that parents confronted with their child's life-threatening disease or near-fatal accident may react with a long-lasting fear of losing their child, even if the immediate threat has disappeared or faded away. Parents' perception of the increased vulnerability of their child could lead to overprotection of the child [35], which might hinder the child in achieving its developmental milestones [36].

\section{Relationship with treatment modality}

Although our group of participants is rather small, we did not find any significant relationship between the need for aftercare in the different domains and treatment modalities. More specifically, we did not find a relationship between the need for neurocognitive aftercare and adjuvant therapy. Learning difficulties have been related to adjuvant therapy earlier (e.g. [4]), although learning difficulties have also been described in survivors with low-grade brain tumors treated with surgery only $[15,16]$.

Our data further indicate that survivors of low-grade tumors treated with surgery only have the same overall need for aftercare compared with those who had adjuvant therapy, which stresses the need for follow-up care. Ris et al. [15] also recommended routine follow-up for children after treatment for low-grade tumors.

\section{Clinical implications and future research}

Our study makes it clear that parents ask for more attention for psychosocial long-term sequelae in childhood brain tumor survivors, regardless of treatment modalities, pointing to the need of a multidisciplinary follow-up screening approach for all brain tumor survivors. In addition, the 
oncologist and the (neuro) psychologist should screen which families are in need for specialized aftercare at particular moments of transition during the development of the child growing in adulthood. This is desirable as survivors run the risk of developing deficits and a substantial number of survivors require special education (31\% compared to $3 \%$ in the normal Dutch population). Further research on evaluating the needs of children and their parents for care should lead to more satisfaction.

Collaboration with school liaisons and the school of the survivor is needed to detect difficulties in time. Moreover, supportive actions can be initiated from the hospital regarding adaptations in primary or secondary education or follow-up training. Patient Reported Outcome (PRO) assessments and internet-based screening tools could be helpful and should be studied on their efficacy in assisting care providers in retrieving the needs for survivors and their parents [37].

Early interventions and preventive psycho-educational programs can improve coping with the long-term sequelae and improve different aspects of quality of life. These interventions directed to the survivors and the parents, include information about programs for improvements for neurocognitive deficits [21-23], empowerment of the child [38] or coaching parents in coping with changes of their child [34]. Additionally, schoolteachers should be informed as well.

\section{Limitations}

Several limitations of the present research should be noted. A major limitation is the use of our survey in which we evaluated the aftercare from a parents' perspective to retrieve the needs for and received aftercare of this group of survivors. A parents' view could add more insight in their perception of improvements of the needs for aftercare, while underestimating or overestimating the needs for aftercare could be influenced by the subjective parental disease experience and could have been prevented by adding more informants [39]. Despite this limitation, our results are in line with previous studies and identify a high need for aftercare in several domains of child functioning and in parenting [1, 9, 15, 19, 34, 35]. Moreover, $82 \%$ of the participants needed special educational services and a substantial number of the children had to transit to special education. This underlines the problems these children have to cope with.

A second limitation is found in the fact we could only tentatively indicate the difference in need for aftercare based on the conditions surgery only or surgery and adjuvant therapy because of our relatively small group in relation to the diversity of patient variables. The influence of radiation therapy could not be evaluated as a separate risk factor. Nevertheless, the high similar need for aftercare in all survivors regardless of the type of treatment indicates that attention for aftercare regarding psychosocial problems and parenting difficulties are a necessity for all brain tumor survivors. Third, the parental evaluation of the received aftercare may be influenced by barriers in the health care system (for instance long waiting lists, long distance to special services) and preexisting psychosocial problems (for instance psychiatric problems in the family, language problems, multiple social problems). In our survey, we did not explore these potential barriers, nor did we explore intrapersonal barriers as mentioned before. More research is needed to explore these barriers in more detail. Fourth, because this is a single center study, representation is limited. Nevertheless, it should be mentioned that to our knowledge this is the first evaluation of the needs for aftercare from a parents' perspective.

Finally, although the survivors were treated in a broad time span (16 years), it should be stated that our center has a long tradition in providing psychosocial aftercare to children treated for cancer, so evaluation of these data from survivors treated at different time points makes sense [40].

\section{Conclusions}

Despite extensive knowledge about the serious long-term sequelae brain tumor survivors could suffer from, this study reveals that increased awareness and a more timely reaction to the need for aftercare is required to meet the need for psychosocial aftercare and parental aftercare more adequately. Treatment centers should focus on a solid followup program by medical and psycho-social staff to perform a timely referral for aftercare in this patient group. Information programs for neurocognitive deficits and psychoeducation about long-term sequelae, strategies aiming at empowerment of the child's autonomy and coping behavior are highly recommended. A well organized co-operation with schools is a prerequisite to meet the needs of survivors and their parents more adequately.

Acknowledgement The authors express their gratitude to the parents of survivors who participated in this study. They also wish to thank Hannah Karst for her help in collecting the data. This study was supported by Dutch foundation KIKA 'Kinderen Kankervrij' (Children Cancerfree).

Conflict of interest statement None of the authors has a conflict of interest to declare in relation to this work.

Open Access This article is distributed under the terms of the Creative Commons Attribution Noncommercial License which permits any noncommercial use, distribution, and reproduction in any medium, provided the original author(s) and source are credited. 


\section{References}

1. Butler RW, Sahler OJ, Askins AM (2008) Interventions to improve neuropsychological functioning in childhood cancer survivors. Dev Disabil Res Rev 14:251-258

2. Butler RW, Mulhern RK (2005) Neurocognitive interventions for children and adolescents surviving cancer. J Pediatr Psychol 30:65-78

3. Moore BD (2005) Neurocognitive outcomes in survivors of childhood cancer. J Pediatr Psychol 30:51-63

4. Mulhern RK, Palmer SL, Merchant TE et al (2005) Neurocognitive consequences of risk-adapted therapy for childhood medulloblastoma. J Clin Oncol 23:5511-5519

5. Geenen MM, Cardoes MC, Kremer L et al (2007) Medical assessment of adverse health outcomes in long-term survivors of childhood cancer. JAMA 297:2705-2715

6. Oeffinger KC, Mertens AC, Sklar CA et al (2006) Chronic health conditions in adult survivors of childhood cancer. N Engl J Med 355:1572-1582

7. Patenaude AF, Kupst MJ (2005) Psychosocial functioning in pediatric cancer. J Pediatr Psychol 30:9-27

8. Bonner MJ, Hardy KK, Wilard VW (2008) Social functioning and facial expression recognition in survivors of pediatric brain tumors. J Pediatr Psychol 33:1142-1152

9. Zebrack BJ, Gurney JG, Oeffinger K et al (2004) Psychological outcomes in long-term survivors of childhood brain cancer: a report from the Childhood Cancer Survivors Study. J Clin Oncol 22:999-1006

10. Eiser C (2007) Beyond survival: quality of life and follow-up after childhood cancer. J Pediatr Psychol 32:1140-1150

11. Stam H, Grootenhuis MA, Last BF (2007) Course of life of survivors of childhood cancer is related to quality of life in young adulthood. J Psychosoc Oncol 25:43-58

12. Peterson CC, Drotar D (2006) Family impact of neurodevelopmental late effects in survivors of pediatric cancer: review of research, clinical evidence, and future directions. Clin Child Psychol Psychiatry 11:349-366

13. Lindahl Norberg A, Lindblad F, Boman KK (2006) Support seeking, perceived support, and anxiety in mothers and fathers after children's cancer treatment. Psycho-Oncology 15:335-343

14. Hutchinson KC, Willard VW, Hardy KK, Bonner MJ (2009) Adjustment of caregivers of pediatric patients with brain tumors: a cross-sectional analysis. Psycho-Oncol 18:515-523

15. Ris MD, Beebe DW, Armstrong FD et al (2008) Cognitive and adaptive outcome in extracerebellar low-grade brain tumors in children: a report from the Children's Oncology Group. J Clin Oncol 26:4765-4770

16. Aarsen FK, Paquier PF, Reddingius RE et al (2006) Functional outcome after low-grade astrocytoma treatment in childhood. Cancer 106:396-402

17. Childhood Cancer Survivorship (2003) Improving care and quality of life. National Cancer Policy Board, Washington

18. Aziz NM, Oeffinger KC, Brooks S et al (2006) Comprehensive long-term follow-up programs for pediatric cancer survivors. Cancer 107:841-848

19. Nathan PC, Patel SK, Dilley K et al (2007) Guidelines for identification of, advocacy for, and intervention in neurocognitive problems in survivors of childhood cancer: a report from the Children's Oncology Group. Arch Pediatr Adolesc Med 161:798-806

20. Wallace WHB, Blacklay A, Eiser C et al (2001) Developing strategies for the long term follow up of survivors of childhood cancer. Br Med J 323:271-274
21. Bowers DC, Adhikari S, El-Khashab YM et al (2009) Survey of long-term follow-up programs in the United States for survivors of childhood brain tumors. Pediatr Blood Cancer 15:1295-1301

22. Butler RW, Copeland DR, Fairclough DL et al (2008) A multicenter, randomized clinical trial of a cognitive remediation program for childhood survivors of a pediatric malignancy. $\mathrm{J}$ Consult Clin Psychol 76:367-378

23. Van't Hooft I, Anderson K, Bergman B et al (2007) Sustained favorable effects of cognitive training in children with acquired brain injury. NeuroRehabilitation 22:109-116

24. Thompson SJ, Leigh L, Christensen R et al (2001) Immediate neurocognitive effects of methylphenidate on learning-impaired survivors of childhood cancer. J Clin Oncol 19:1802-1808

25. Kazak AE (2005) Evidence based interventions for survivors of childhood cancer and their families. J Pediatr Psychol 30:2939

26. Last BF, Stam H, Onland-van Nieuwenhuizen AM, Grootenhuis MA (2007) Positive effects of a psycho-educational group intervention for children with a chronic disease: first results. Patient Educ Couns 651:101-112

27. Barrera M, Schulte F (2009) A group social skills intervention program for survivors of childhood brain tumors. J Pediatr Psychol 34:1108-1118

28. Recklitis CJ, Lockwood RA, Rothewell MA et al (2006) Suicidal ideation and attempts in adult survivors of childhood cancer. J Clin Oncol 24:3852-3857

29. Baile WF, Aaron J (2005) Patient-physician communication in oncology: past, present, and future. Curr Opin Oncol 17:331335

30. Lazarus RS (1983) The costs and benefits of denial. In: Breznitz S (ed) The denial of stress. International Universities Press, New York, pp 1-30

31. Oeffinger KC, Wallace WH (2006) Barriers to follow-up care of survivors in the United States and the United Kingdom. Ped Blood Cancer 46:135-142

32. Hewitt M, Greenfield S, Stovall E (2005) From cancer patient to cancer survivor: lost in transition. The National Press, Washington

33. Bredart A, Bouleuc C, Dolbeault S (2005) Doctor-patient communication and satisfaction with care in oncology. Curr Opin Oncol 17:351-354

34. Lindahl Norberg A, Steneby S (2009) Experiences of parents of children surviving brain tumour: a happy ending and a rough beginning. Eur J Cancer Care 18:371-380

35. Coletti CJM, Wolfe-Christensen C, Carpentier MY et al (2008) The relationship of parental overprotection, perceived vulnerability, and parenting stress to behavioral, emotional, and social adjustment in children with cancer. Pediatr Blood Cancer 51:269-274

36. Stam H, Grootenhuis MA, Last BF (2005) The course of life of survivors of childhood cancer. Psycho-Oncology 14:227-238

37. McDowell ME, Occhipinti S, Ferguson M et al (2009) Predictors of change in unmet supportive care needs in cancer. PsychoOncology July 14th: Epub ahead of print

38. Maurice-Stam H, Silberbusch LM, Last BF et al (2009) Evaluation of a psycho-educational group intervention for children treated for cancer: a descriptive pilot study. PsychoOncology 18:762-766

39. Majnemer A, Shevell M, Law M et al (2008) Reliability in the ratings of quality of life between parents and their children of school age with cerebral palsy. Qual Life Res 9:1163-1171

40. Langeveld (2003) Cured of cancer. From childhood to adulthood quality of survival. Dissertation, University of Amsterdam 